\title{
A rare case of adenomatoid tumour presented as back pain: case report and brief review of the literature
}

\begin{abstract}
Adenomatoid tumours of mesothelian origin are rare neoplasms. The incidences of these findings make difficult the differential diagnosis in most cases. The newest evidence obtained from the ultrastructural and immunohistochemical findings indicate that adenomatoid tumours are of a mesothelial nature, although differential diagnosis is challenging, as in the case of a $49 \mathrm{yr}$ male presented with cough and back pain.
\end{abstract}

Keywords: adenomatoid tumour, rare neoplasms, adrenal gland
Volume 6 Issue 3 - 2018

\section{Charalampos Massouras, Andromachi Vryonidou, George Sakorafas, Sofia Simopoulou, Nicholaos Barouchos, loanna Markaki, Christos Mpalaskas \\ Private Office, University of Agriculture, Athens Medical Center of Peristeri, Red Cross Hospital, Agios Savvas Hospital, Greece}

Correspondence: Charalampos Massouras, Private Office, University of Agriculture, Athens Medical Center of Peristeri, Red Cross Hospital, Agios Savvas Hospital, 36, Stravonos Str Peristeri,Attica, Greece, Email endomx@gmail.com

Received: January 02, 2018 | Published: June 13,2017

\section{Background}

Adenomatoid tumours are presented as rare neoplasms of mesothelian origin, with different location, which can easily mimic specific diseases presented as adrenal incidantelomas, eg pheochromytoma, Cushing syndrome or adrenal carcinoma. The incidence does not allow providing specific pathognomic characteristics and the differential diagnosis may be proven challenging.

\section{Case presentation}

NK, a 49 year old mechanic engineer, without remarkable medical history, non smoker, was examined by cardiologist (IM) \& pneumologist $(\mathrm{NB})$ due to atypical chest \& mostly back pain., after having visited public hospital (2th 2017). Where blood count, troponine, biochemical analysis, chest Xray \& heart doppler revealed anything notable. The chest ct scan ordered by NB revealed multiple shadings up to $3 \mathrm{~mm}$, propably as remains of lung infection. The main finding was a large hypodense mass $8 * 4 \mathrm{~cm}$ at the right adrenal location, probably outrived from it.

\section{Management}

Referral to endocrinologist (CM) was followed. By the clinical examination a slight increase of blood pressure $(155 / 90 \mathrm{mmHg})$ was obtained. The patient did not complain for fatigue, weight loss, tachycardia, palpations or perspirations and the findings wher consistent. Hormonal measurements \& specific adrenal ct scan was ordered. The first revealed any oversecretion of cortisole, metanephrines or VMA [UFC $45.5 \mathrm{mcg} / \mathrm{g}$ CR, LC/MSMS, MNM 95(52-341) $\mu \mathrm{g} / 24 \mathrm{~h}, \mathrm{NM}$ 293(88-444) $\mu \mathrm{g} / 244$, VMA 4.1(1.8-6.7 $\mathrm{mg} / 24 \mathrm{~h})]$. The thyroid function, the hypothalamic hypophysis adrenal axis and the aldosterone-renin ration proved within normal ranges. The overnight suppression test (1mg dexamethasone) showed slide oversecretion (2.0) Nevertheless, the low dosage dexamethasone suppression test was negative for secretion (F:0.6) Free plasma normetanephrines \& metanephrines (Mayo Clinic) revealed any abnormal results. VMA was repeated \& proven within normal ranges at the second measurement.

An expert opinion was kindly given by AV, who recommended surgical removal due to the characteristics, dimension \& hormonal measurements of the mass.

\section{Radiologic findings}

A localized specific adrenal CT scan revealed a hypodense, clear delimited mass of the right adrenal gland, with dimensions of $8 \times 4 \times 6,6$ $\mathrm{cm}$, without contrast enhancement, measured of 11 Housefield units., mainly characterized by the radiologist as adenoma see Figure 1.

\section{Surgical procedure}

MR NM was consulted by GS, who operated the patient by open technique $(04 / 05 / 2017)$, without any serious postsurgical complication, other than a mild lung infection which recovered easily after antibiotic treatment. The whole procedure durated three days

\section{Histopathologic examination}

The findings where constinent of a large eggsize tumour, weight $95 \mathrm{gr}$, dimension of $8 \times 6,5 \times 1,8 \mathrm{~cm}$, with smooth external surface. The epitheliods cells are in some locations wasllowed in dense stroma of inotic places. The immunochemistry revealed CK $27+$, Vim (+), Calretinin(=), Chromogranin (-), CK20(-), findings which represent mostly adenomatoid tumour of right adrenal gland (Figure 2).

Mr NK, was reassured that the mass, although seemed as of adrenal origin was categorized as adenomatoid tumour, meaning that the prognosis, after the removal may be better than (eg) of an adrenal carcinoma $\&$ the possibility of malignancy remains low.

The patient reexamined 6 mo later, where the adrenal axis has been examined through act suppression test, with normal response of cortisole $(\mathrm{F} 30 \mathrm{~min} 22.2 \mu \mathrm{g} / \mathrm{dl})$, meaning that no need of replacement is required (Figure 3) (Figure 4). 


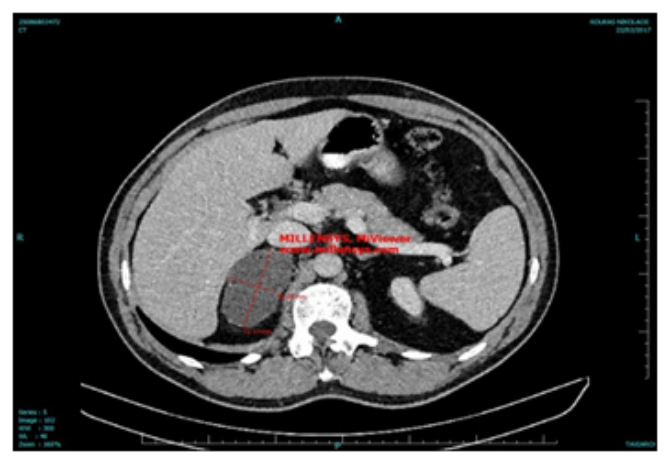

Figure I Radiologic Findings A localized specific adrenal CT scan.

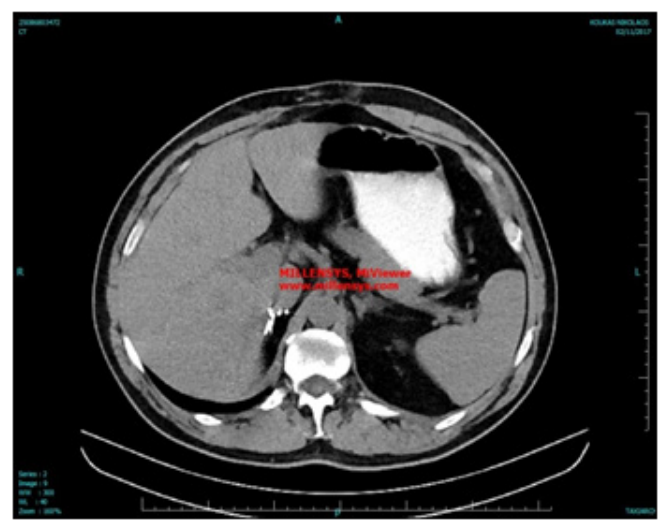

Figure $3 \mathrm{New}$ adrenal CT scan has been obtained which showed excellent postsurgical results.

\section{Discussion}

Adrenal adenomatoid tumors are derived from mesothelial rests in the adrenal gland., ${ }^{1,2}$ During embryogenesis there is close relationship between the adrenal glands and the Mullerian tract, so mesothelial rests could be present in this unexpected site such as the adrenal gland. These tumors are mostly nonfunctional, asymptomatic and discovered incidentally, during radiological examinations, surgery or on autopsy. Adenomatoid tumors are not associated with diabetes, but seven cases were found to be associated with hypertension. ${ }^{3}$

\section{Acknowledgments}

None.

\section{Conflict of interest}

No conflicts of interest. Informed written consent was obtained by the patient.

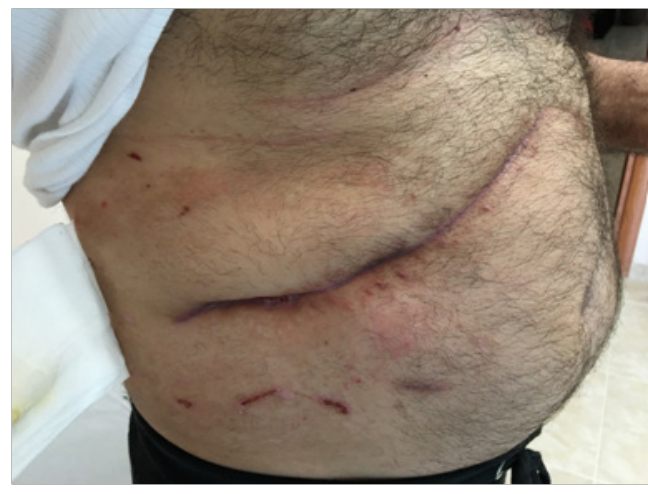

Figure $\mathbf{2}$ The postsurgical findings can be shown in the next image.

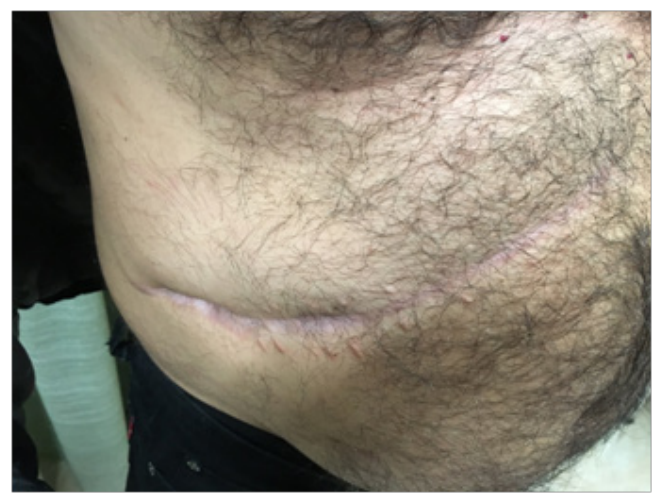

Figure 4 Post surgical insection shows sufficient healing as in the image below.

\section{References}

1. Sağlican Y, Kurtulmus N, Tunca F, et al. Mesothelial derived adenomatoid tumour in a location devoid of mesothelium: adrenal adenomatoid tumour. BMJ Case Rep. 2015; pii: bcr2015211147.

2. El-Daly H, Rao P, Palazzo F, et al. A Rare Entity of an Unusual Site: Adenomatoid Tumour of the Adrenal Gland: A Case Report and Review of the Literature. Pathology Research International. 2010. ID: 702472.

3. Krstevska B, Mishevska SJ, Jovanovic R. Adenomatoid Tumor of the Adrenal Gland in Young Woman: From Clinical and Radiological to Pathological Study. Rare Tumors. 2016;8(4):6506. 